Schroeder-Wildberg

Entscheidungs- und Lernverhalten an Wertpapiermärkten 
GABLER EDITION WISSENSCHAFT 
Uwe Schroeder-Wildberg

\section{Entscheidungs- und Lernverhalten an Wertpapiermärkten}

Psychologische Aspekte von Börsenentscheidungen

Mit einem Geleitwort

von Prof. Dr. Wolfgang Gerke

Springer Fachmedien Wiesbaden GmbH 
Die Deutsche Bibliothek - CIP-Einheitsaufnahme

\section{Schroeder-Wildberg, Uwe:}

Entscheidungs- und Lernverhalten an Wertpapiermärkten :

psychologische Aspekte von Börsenentscheidungen / Uwe Schroeder-Wildberg.

Mit einem Geleitw. von Wolfgang Gerke.

- Wiesbaden : Dt. Univ.-Verl.; Wiesbaden : Gabler, 1998

(Gabler Edition Wissenschaft)

Zugl.: Erlangen - Nürnberg, Univ., Diss., 1997

ISBN 978-3-8244-6552-1

DOI 10.1007/978-3-663-08457-0

ISBN 978-3-663-08457-0 (eBook)

n2

Alle Rechte vorbehalten

Gabler Verlag, Deutscher Universitüts-Verlag, Wiesbaden

(C) Springer Fachmedien Wiesbaden 1998

Ursprünglich erschienen bei Betriebswirtschaftlicher Verlag

Dr. Th. Gabler GmbH, Wiesbaden 1998

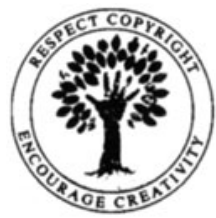

Das Werk einschließlich aller seiner Teile ist urheberrechtlich geschützt. Jede Verwertung außerhalb der engen Grenzen des Urheberrechisgesetzes ist ohne Zustimmung des Verlages unzulässig und strafbar. Das gilt insbesondere für Vervielfältigungen, Übersetzungen, Mikroverfilmungen und die Einspeicherung und Verarbeitung in elektronischen Systemen.

hitp://www.gabler-online.de

Höchste inhaltliche und technische Qualität unserer Produkte ist unser Ziel. Bei der Produktion und Auslieferung unserer Bücher wollen wir die Umwelt schonen: Dieses Buch ist auf säurefreiem und chlorfrei gebleichtem Papier gedruckt.

Die Wiedergabe von Gebrauchsnamen, Handelsnamen, Warenbezeichnungen usw. in diesem Werk berechtigt auch ohne besondere Kennzeichnung nicht zu der Annahme, daß solche Namen im Sinne der Warenzeichen- und Markenschutz-Gesetzgebung als frei zu betrachten wären und daher von jedermann benutzt werden dürften. 


\section{Geleitwort}

Neben theoretischen und empirischen Untersuchungen zur Kapitalmarkttheorie, gewinnen zunehmend auch experimentelle Erklärungsversuche des Verhaltens an Kapitalmärkten an Bedeutung. Am Lehrstuhl für Bank- und Börsenwesen der Universität Erlangen-Nürnberg wurde deshalb ein komplexes Börsenhandelssystem entwickelt, mit dem auf computergestützter Basis Entscheidungssituationen an Kapitalmärkten experimentell simuliert werden können. Die Experimentteilnehmer erhalten dabei die Möglichkeit, Gelder in Festgeld anzulegen, Kredite aufzunehmen, durch ihre Eingabe von Geld- und Briefkursen Aktien zu kaufen und zu verkaufen sowie umfangreiche Informationen über Risiko- und Renditedaten abzurufen. Abweichend von einigen spieltheoretischen Ansätzen und Lotterieexperimenten werden die entwickelten Börsenexperimente bewußt komplex gehalten, um die Vielfalt der realen Entscheidungssituationen möglichst naturgetreu abzubilden. Hierdurch werden einerseits Assoziationen der Entscheider mit realen Entscheidungssituationen gefördert, andererseits aber auch die isolierten Analysen einzelner Effekte erschwert.

In der vorliegenden Arbeit wird die experimentelle Computerbörse genutzt, um das Individualverhalten der Teilnehmer an den Börsenmärkten zu untersuchen. Während in der Kapitalmarkttheorie primär aggregierten Daten die größte Aufmerksamkeit zugewendet wird, konzentriert sich die Arbeit auf individuelle Lernprozessen und Entscheidungsmuster. Sie wird dabei von der These geleitet, daß sich insbesondere auf der Individualebene ein wesentlich stärkeres Abweichen von rationalem Entscheidungsverhalten beobachten läßt, als dies in den theoretischen Modellen auf der Makroebene angenommen wird. Bereits die menschliche Informationsverarbeitungskapazität zwingt die Entscheider, komplexe Probleme zu vereinfachen. Dies gilt insbesondere für Entscheidungen unter Zeitdruck, wie sie an den Kapitalmärkten häufig gegeben sind. Die begrenzte menschliche Informationskapazität führt zu unterschiedlichen Umweltinterpretationen und damit auch zu Wertpapierumsätzen aufgrund heterogener Erwartungen.

Abweichungen vom rationalen Entscheidungsverhalten können auch darauf zurückzuführen sein, daß die Entscheider das objektive oder illusionäre Gefühl einer Handlungskontrolle wahrnehmen. Die kann sich in den Experimenten in Versuchen der Marktbeeinflussung widerspiegeln. Beim Gefühl fehlender Handlungskontrolle wird häufig Streßempfinden hervorgerufen, durch das gegebenenfalls Kurssteigerungen oder Kurseinbrüche verstärkt werden. Daneben beeinflussen Stimmungen das Entscheidungsverhalten an den Börsenmärkten. So hat die Stimmungslage des Individuums Rückwirkungen auf seine Risikowahrnehmung. 
Zur Analyse des Individualverhaltens wird in dieser Arbeit mit Hilfe der experimentellen Computerbörse das Entscheidungs- und Lernverhalten von Probanden in bezug auf ihre Informationsverarbeitung, Preisbildung und ihr Risikoverhalten analysiert. Differenziert wird hierzu zwischen Experimenten, in denen die Probanden keine Experimenterfahrung aufweisen und Experimenten, in denen die Probanden zuvor an mindestens einem gleichgearteten Experimentverlauf teilgenommen haben. Die Arbeit bietet neue Einblicke in individuelle Entscheidungsprozesse an den Wertpapiermärkten, ihre Beeinflussung durch Erfahrungswerte, soziale Effekte, Stimmungen und Wahrnehmung der Handlungskontrolle. Es wird überzeugend aufgezeigt, daß in der experimentellen Forschung Lernprozesse der Probanden eine erhebliche Rolle spielen und daß insbesondere auf Individualebene höchstens beschränkt rationales Verhalten zu beobachten ist. Für die weitere experimentelle Kapitalmarktforschung liefert die Arbeit wichtige Hinweise.

Prof. Dr. Wolfgang Gerke 


\section{Vorwort}

Die vorliegende Arbeit wurde im Februar 1997 von der wirtschaftswissenschaftlichen Fakultät der Universität Erlangen-Nürnberg als Dissertation angenommen. Meinem akademischen Lehrer, Herrn Prof. Dr. Wolfgang Gerke, danke ich herzlichst für die persönliche Betreuung und Diskussionsbereitschaft während und nach meiner Zeit am Lehrstuhl. Herrn Prof. Dr. Dieter Euler danke ich für Übernahme des Zweitgutachtens und das Interesse am interdisziplinären Ansatz der Arbeit.

Meinen ehemaligen Kollegen am Lehrstuhl für Bankbetriebslehre in Mannheim und später am Lehrstuhl für Bank- und Börsenwesen in Nürnberg danke ich für die gemeinsame Zeit, die freundschaftliche Atmosphäre und die Diskussions- und Hilfsbereitschaft. Besonderer Dank gilt meinem Freund und ehemaligen Kollegen Herrn Dr. Horst Bienert für viele, über das zu erwartende Maß hinausgehende Beiträge sowie zahllose fruchtbare und humorvolle Diskussionsstunden, die den beschwerlichen Weg der Erkenntnis erleichtert haben. Ein besonderer Dank gilt auch meinen Eltern und allen Freunden für ihre Anteilnahme, Unterstützung und ihr Verständnis.

Nicht angemessen in Worte zu fassen ist schließlich der Dank an meine Frau Heide, die nicht nur auf viele gemeinsame Stunden verzichten mußte, sondern mir mit ihrem Verständnis, ihrer Fürsorge und ihrem positiven Wesen die Kraft gegeben hat, auch die harten Stunden der Promotionszeit zu bewältigen. 


\section{Inhaltsverzeichnis}

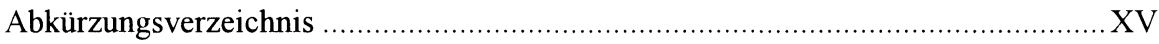

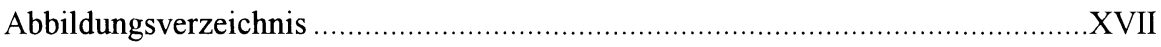

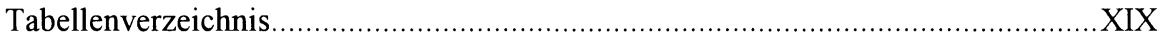

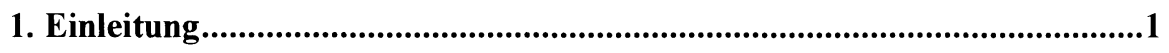

1.1 Problemstellung und Abgrenzung des Themas ...............................................

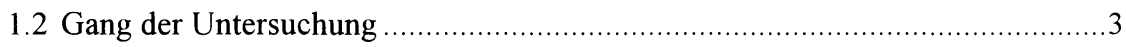

2. Entscheidungsverhalten an Wertpapiermärkten ..........................................

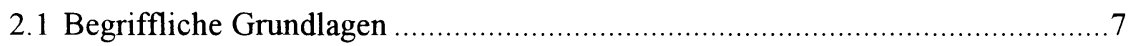

2.2 Merkmale von Entscheidungen an Wertpapiermärkten ................................

2.3 Entscheidungen an Wertpapiermärkten aus normativer Perspektive ..................11

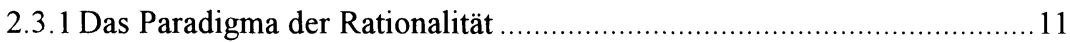

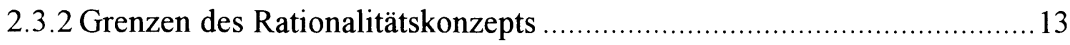

2.3.2.1 Anforderungen realer Entscheidungssituationen ....................... 13

2.3.2.2 Entscheidungsverhalten in einfachen Wahlsituationen ................ 14

2.3.2.2.1 Methodische Beurteilung ............................. 14

2.3.2.2.2 Experimentelle Ergebnisse ............................. 18

2.3.3 Zur Rationalitätsfunktion der Marktinstitution.....................................21

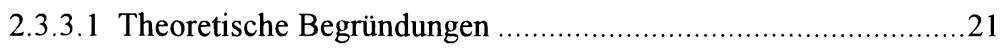

2.3.3.1.1 Die kausale Position.....................................22

2.3.3.1.2 Die positivistische Position ............................24

2.3.3.2 Modellierung des Entscheidungsverhaltens im

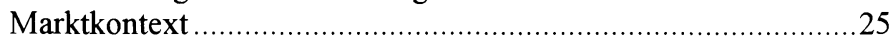

2.3.3.2.1 Rationale Erwartungen .................................25 
2.3.3.2.2 Portefeuilleentscheidungen............................28

2.3.3.3 Empirische Überprüfung von Marktprozessen ............................30

2.3.3.4 Experimentelle Untersuchungen von Marktprozessen ................ 32

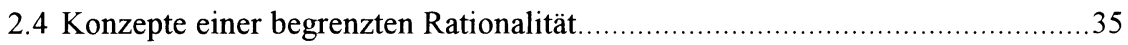

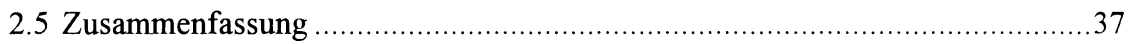

3. Die experimentelle Computerbörse CAT ...............................................39

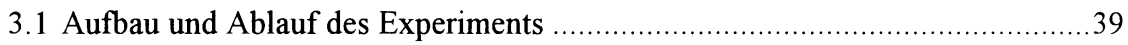

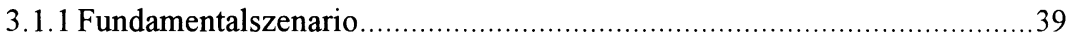

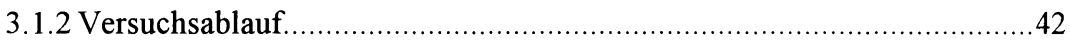

3.1.3 Aktienhandel und Kursermittlungsprinzip ........................................4 44

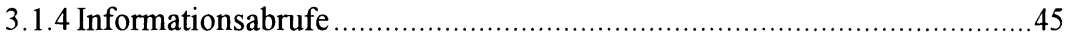

3.2 Die Entscheidungssituation der Marktteilnehmer .........................................47

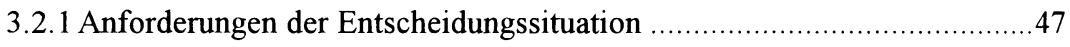

3.2.2 Strategische Aspekte der Orderlimitierung .......................................49

\section{Ein verhaltensorientierter Ansatz zur Erklärung des} Entscheidungsverhaltens an einem Wertpapiermarkt ...............................51

4.1 Allgemeine Charakteristika von Entscheidungsprozessen .............................51

4.2 Determinanten von Entscheidungsprozessen ..........................................54

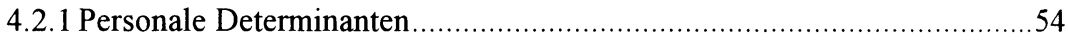

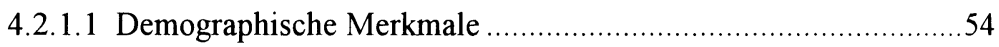

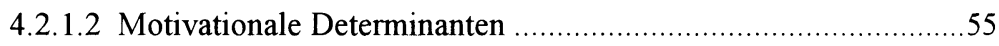

4.2.1.2.1 Leistungsmotivation im Entscheidungs-

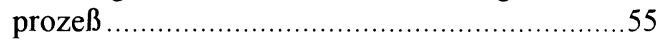

4.2.1.2.2 Motivationstheoretische Erklärung aggregierter Marktprozesse ............................60

4.2.1.3 Handlungskontrolle 63 
4.2.1.3.1 Das kontrolltheoretische Grundkonzept 63

4.2.1.3.2 Handlungskontrolle und Attribuierung 67

4.2.1.4 Stimmungen

4.2.1.5 Kognitive Strukturiertheit

4.2.2 Situative Determinanten .72

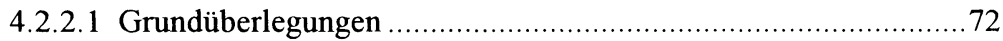

4.2.2.2 Komplexität der Entscheidungssituation ..................................73

4.2.2.3 Zeitliche Aspekte des Entscheidungsprozesses ..........................76

4.2.2.4 Zufalls- und Aktionsabhängigkeit der Entscheidungskonsequenzen. 77

4.2.2.5 Bedeutung früherer Entscheidungen ...................................... 79

4.2.2.6 Selbstverpflichtung durch eine Entscheidung ........................... 81

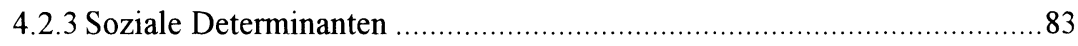

4.2.3.1 Grundüberlegungen zur Sozialität des Marktes ..........................83

4.2.3.2 Marktspezifische Sozialisation über

Entscheidungsinterdependenzen und Interaktionen ...................85

4.3 Kognitive Prozesse bei Entscheidungen an Wertpapiermärkten ......................90

4.3.1 Kognitive Prozesse der Informationsaufnahme .....................................90

4.3.1.1 Qualitative Aspekte der Informationsaufnahme ........................90

4.3.1.2 Quantitative Aspekte der Informationsaufnahme.......................95

4.3.1.2.1 Kapazitätsgrenzen der Informations-

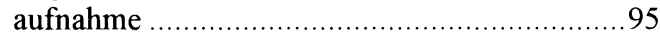

4.3.1.2.2 Anpassungsmechanismen bei Informationsüberlastung 98

4.3.1.3 Auswirkungen der Informationspräsentation und -verfügbarkeit

4.3.2 Kognitive Prozesse der Informationsverarbeitung ................................ 101

4.3.2.1 Grundüberlegungen zur Informationsverarbeitung ................... 101

4.3.2.2 Erwartungen als zentraler Bestandteil der Informationsverarbeitung. 
4.3.2.2.1 Bildung von Erwartungen.............................. 103

4.3.2.2.2 Erwartungen und Trendanalyse .................... 105

4.3.2.3 Anspruchsniveaus als Orientierungsmaßstab im

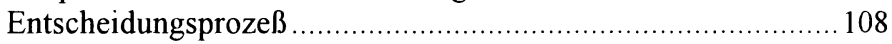

4.3.2.3.1 Grundsätzliche Überlegungen zum Anspruchsniveau ........................................ 108

4.3.2.3.2 Anspruchsniveauanpassung im Marktkontext .......................................... 110

4.3.2.3.3 Modellierung idealtypischer Entscheidungssituationen.

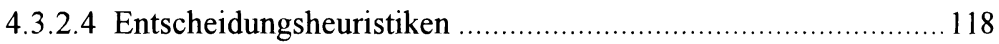

4.3.2.5 Verhaltenseffekte im Informationsverarbeitungsprozeß ........... 120

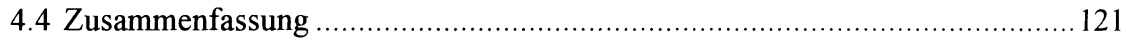

5. Lernprozesse bei Entscheidungen an Wertpapiermärkten .....................125

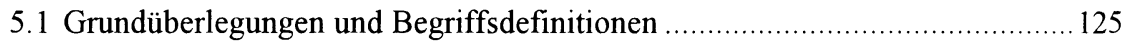

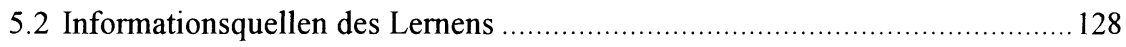

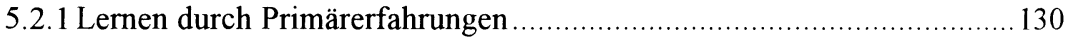

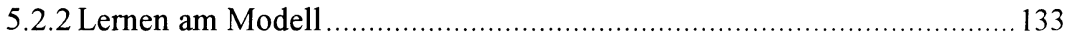

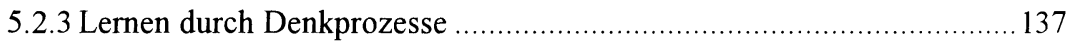

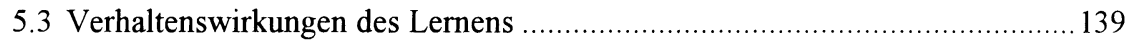

5.3.1 Die Bildung nicht-kognitiver Handlungsstrukturen..............................139

5.3.2 Die Bildung kognitiver Handlungsstrukturen ....................................... 144

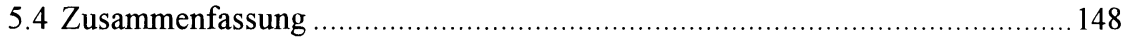

6. Entscheidungs- und Lernverhalten im Marktexperiment ........................151

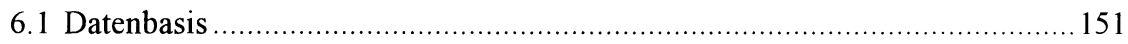

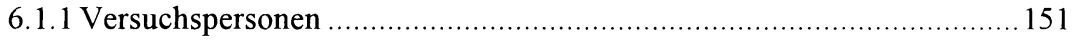




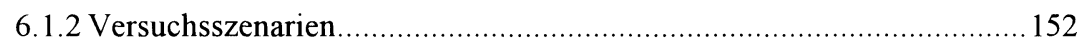

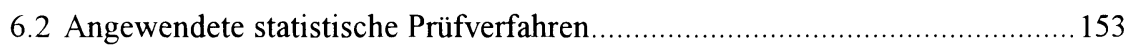

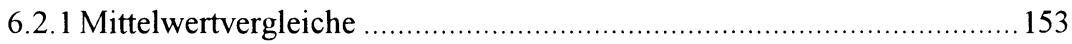

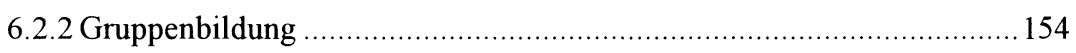

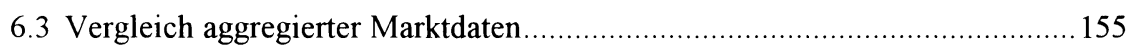

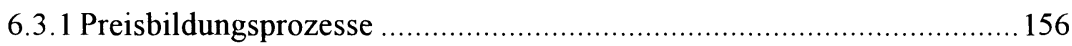

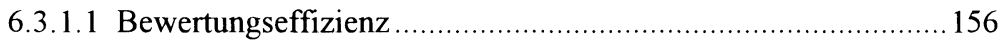

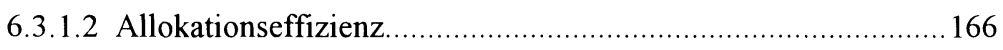

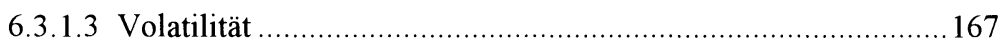

6.3.2 Ordereingaben und Transaktionen ....................................................... 170

6.3.2.1 Häufigkeit von Ordereingaben und Transaktionen ................... 170

6.3.2.2 Erwartungsheuristiken und Ordereingaben ............................ 171

6.3.2.3 Strategische Aspekte der Orderlimitierung............................. 172

6.3.2.4 Prominenzstrukturen bei der Orderlimitierung ........................ 177

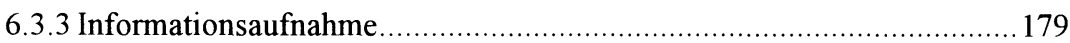

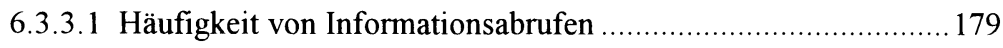

6.3.3.2 Entscheidungskonsequenzen von Informationsabfragen........... 185

6.4 Entscheidungs- und Lernverhalten auf der Individualebene ......................... 188

6.4.1 Entscheidungsverhalten aus der Perspektive der Marktteilnehmer ......... 188

6.4.2 Verhaltensunterschiede im Entscheidungsprozeß ............................... 191

6.4.2.1 Klassifizierung von Entscheidungsträgern ............................ 191

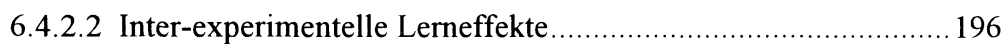

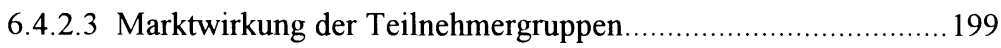

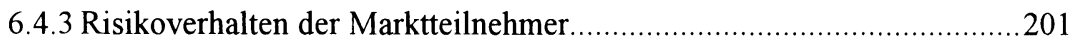

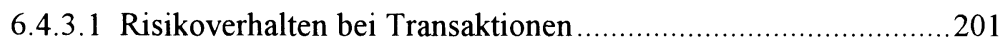

6.4.3.2 Risikoverhalten bei der Portefeuillebildung ...........................203

6.4.4 Anspruchsniveauanpassung im Marktkontext .....................................206 
6.4.4.1 Anpassungsprozesse nach Transaktionen ...............................206

6.4.4.2 Anpassungsprozesse nach erfolglosen Ordereingaben..............209

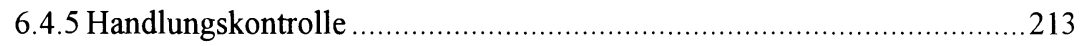

6.4.6 Verhaltenskonsequenzen früherer Entscheidungen ............................219

6.4.7 Selbstverpflichtung durch Entscheidungen ....................................223

7. Zusammenfassung der zentralen Ergebnisse ........................................227

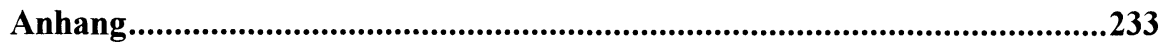

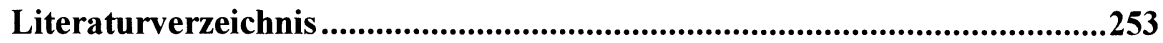




\section{Abkürzungsverzeichnis}

\begin{tabular}{ll} 
Abb. & Abbildung \\
AER & American Economic Review \\
Aufl. & Auflage \\
Bd. & Band \\
bzw. & beziehungsweise \\
ca. & circa \\
DBW & Die Betriebswirtschaft \\
d.h. & das heißt \\
Diss. & Dissertation \\
ed. & edited \\
et al. & und andere \\
etc. & et cetera \\
Habil. & Habilitation \\
Hrsg. & Herausgeber \\
hrsg. v. & herausgegeben von \\
Jg. & Jahrgang \\
JoF & Journal of Finance \\
No. & Nummer \\
Nr. & Nummer \\
o.V. & ohne Verfasser \\
S. & Seite \\
Tab. & Tabelle \\
u.a. & unter anderem \\
vgl. & vergleiche \\
\hline . &
\end{tabular}


Vol. Volume

z.B. zum Beispiel

ZBB Zeitschrift für Bankrecht und Bankwirtschaft

ZfB Zeitschrift für Betriebswirtschaft 


\section{Abbildungsverzeichnis}

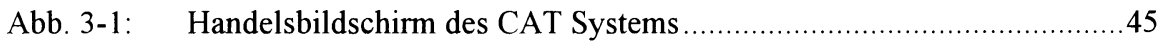

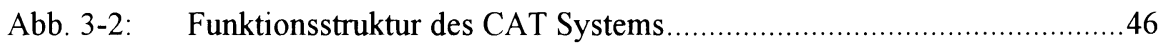

Abb. 4-1: Multiple Anspruchsniveaus in einer Verhandlungssituation ................114

Abb. 4-2: Anspruchsniveaus und Bestandsbewertung …....................................117

Abb. 6-1: Marktindizes im Vergleich mit hypothetischen Kursen (Exp. A1/A2) . 157

Abb. 6-2: Marktindizes im Vergleich mit hypothetischen Kursen (Exp. A3/A4) . 158

Abb. 6-3: Marktindizes im Vergleich mit hypothetischen Kursen (Exp. B1/B2).. 159

Abb. 6-4: Marktindizes im Vergleich mit hypothetischen Kursen (Exp. B3/B4).. 160

Abb. 6-5: Streudiagramm der Gewinnerwartungen und

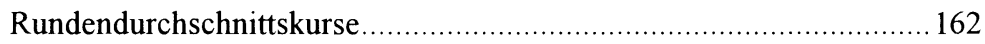

Abb. 6-6: Streudiagramm der Gewinnerwartungen und

Rundendurchschnittskurse (Exp. A1-A4) ....................................... 163

Abb. 6-7: Anpassung der Rundendurchschnittskurse an Gewinnänderungen....... 164

Abb. 6-8: Durchschnittlicher Anteil des Kontostandes am Gesamtvermögen

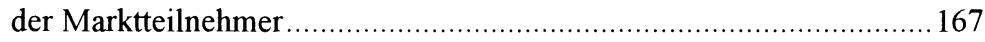

Abb. 6-9: Anteil der Ordereingaben bei Gewinnsequenzen (Exp. A1-A4) ...........171

Abb. 6-10: Anteil der Ordereingaben bei Gewinnsequenzen (Exp. B1-B4) ...........172

Abb. 6-11: Informationsabrufe im Experimentverlauf ....................................... 182

Abb. 6-12: Informationsabrufe Geld-Brief-Spanne im Experimentverlauf............. 183

Abb. 6-13: Clusterwechsel in Abhängigkeit vom Tradingerfolg ......................... 198

Abb. 6-14: Rendite-Risiko-Strukturen je Teilnehmer .........................................205 


\section{XVIII}

Abb. 6-15: Kauforderlimitierung bei positiven Gewinnsequenzen (Exp. A1-A4) ..220

Abb. 6-16: Kauforderlimitierung bei positiven Gewinnsequenzen (Exp. B1-B4) ..220

Abb. A-1: Marktindizes und Kursverläufe im Vergleich mit hypothetischen Kursen (Exp. A1/A2) 243

Abb. A-2: Marktindizes und Kursverläufe im Vergleich mit hypothetischen Kursen (Exp. A3/A4) 245

Abb. A-3: Marktindizes und Kursverläufe im Vergleich mit hypothetischen Kursen (Exp. B1/B2) 247

Abb. A-4: Marktindizes und Kursverläufe im Vergleich mit hypothetischen Kursen (Exp. B3/B4) 249 


\section{Tabellenverzeichnis}

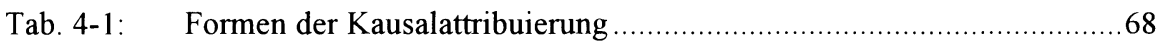

Tab. 4-2: Potentielle Reihenfolge der Informationsabfragen im CAT-System..... 100

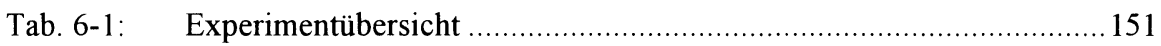

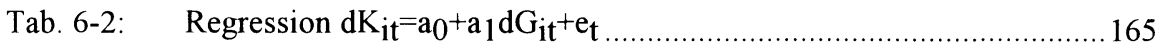

Tab. 6-3: Kursanpassung bei sinkenden und steigenden Gewinnerwartungen ...... 165

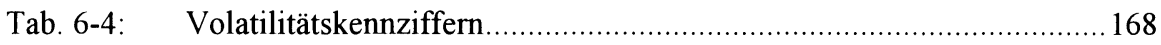

Tab. 6-5: Volatilitätskennziffern Experimente A1 bis A4 ................................. 169

Tab. 6-6: Volatilitätskennziffern Experimente B1 bis B4 ................................. 169

Tab. 6-7: Orders, Transaktionen, Stornierungen je Titel in einer Handelsrunde .. 170

Tab. 6-8: Prozentuale Differenz eingegebener Orders zur Marktgegenseite ........ 173

Tab 6-9: Prozentuale Differenz eingegebener Limitorders zur

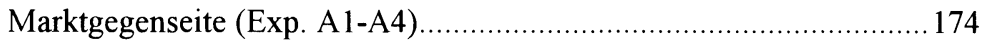

Tab. 6-10: Prozentuale Differenz eingegebener Limitorders zur

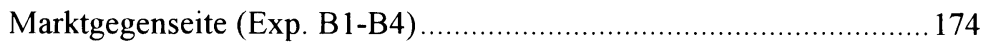

Tab 6-11: Prozentuale Differenz eingegebener Orders zur letzten Kursnotierung

Tab. 6-12: Prozentuale Differenz eingegebener Orders zur letzten

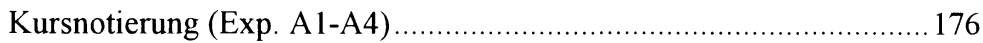

Tab. 6-13: Prozentuale Differenz eingegebener Orders zur letzten

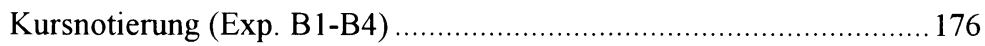

Tab. 6-14: Prominenzstruktur Stück je Order (Exp. A1-A4) .............................. 178

Tab. 6-15: Prominenzstruktur Stück je Order (Exp. B1-B4) ……....................... 178 
Tab. 6-16: Informationsabfragen je Teilnehmer in einer Handelsrunde.

Tab. 6-17: Informationsabfragen je Teilnehmer in einer Handelsrunde (Exp. A1-A4)

Tab 6-18: Informationsabfragen je Teilnehmer in einer Handelsrunde (Exp. B1-B4)

Tab. 6-19: Häufigkeiten von Ordereingaben in verschiedenen Zeitspannen nach dem Abruf der Geld-Brief Spannen

Tab. 6-20: Orderabstand zu Marktgegenseite innerhalb von 30 Sekunden nach Abruf der Geld-Brief Spannen

Tab. 6-21: Orderabstand zu eigenen Marktseite innerhalb von 30 Sekunden nach Abruf der Geld-Brief Spannen

Tab. 6-22: Clusteranalyse für Experimente A1-A4

Tab. 6-23: Einzelwerte für Gruppe 3 .

Tab. 6-24: Clusteranalyse für Experimente B1-B4

Tab. 6-25: Clusterzugehörigkeit in 1. und 2. Serie

Tab. 6-26: Verteilung der Gruppen über Experimente A1 bis A4

Tab. 6-27: Verteilung der Gruppen über Experimente B1 bis B4 200

Tab. 6-28: Durchschnittliches KGV je Transaktionen 201

Tab. 6-29: Durchschnittliche Rendite- $u$. Risikokennziffern der Aktienportefeuilles. 203

Tab. 6-30: Durchschnittliche Rendite- u. Risikokennziffern der Gesamtportefeuilles 204

Tab. 6-31: Verteilungskennziffern Risiko-Rendite-Strukturen 204

Tab. 6-32: Anspruchsniveauanpassung nach Transaktionen 207 
Tab. 6-33: Anspruchsniveauanpassung bei Ordersequenzen

Tab. 6-34: Klassifizierung nach Handlungskontrolle

Tab. 6-35: Primäre Handlungskontrolle und Risikoverhalten (Exp. A1-A4) .........215

Tab. 6-36: Primäre Handlungskontrolle und Risikoverhalten (Exp. B1-B4)..........215

Tab. 6-37: Handlungskontrolle und Orderlimitierung ab elfter Handelsrunde (Exp. A1-A4)

Tab. 6-38: Handlungskontrolle und Orderlimitierung ab elfter Handelsrunde (Exp. B1-B4)

Tab. 6-39: Handlungskontrolle und Orderlimitierung bei Aktien mit negativer Gewinnerwartung. 218

Tab. 6-40: Entscheidungsverhalten bei Mißerfolgen (Teil I). .222

Tab. 6-41: Entscheidungsverhalten bei Mißerfolgen (Teil II). 223

Tab. 6-42: Risikobereitschaft und Ordergröße 224

Tab. A-1: Prominenzstruktur Orderlimitierung (Exp. A1-A4)... .251

Tab. A-2: Prominenzstruktur Orderlimitierung (Exp. B1-B4) .251 